Revista Arbitrada Interdisciplinaria de Ciencias de la Salud. SALUD Y VIDA

Volumen 3. Número 6. Año 3. Julio - Diciembre 2019

Hecho el depósito de Ley: FA2016000010 ISSN: 2610-8038

FUNDACIÓN KOINONIA (F.K)

Santa Ana de Coro, Venezuela.

Carmen Silverio Calderón; Cristhian Damian Cabrera Gia

DOI: http://dx.doi.org/10.35381/s.v.v3i6.309

\title{
Control de las medidas de asepsia en el área neonatológica de un Hospital al sur de Ecuador
}

\section{Control of aseptic measures in the neonatological area of a Hospital in southern Ecuador}

\author{
Carmen Elisabeth Silverio Calderón \\ csilverio@umachala.edu.ec \\ Universidad Técnica de Machala \\ Ecuador \\ https://orcid.org/0000-0003-3587-4149 \\ Cristhian Damian Cabrera Gia \\ ccabreragia@gmail.com \\ Universidad Técnica de Machala \\ Ecuador \\ https://orcid.org/0000-0003-3582-7545
}

Recibido: 09 de abril de 2019

Aprobado: 20 de mayo de 2019

\section{RESUMEN}

El presente artículo es un estudio descriptivo que cumple con el objetivo de identificar la aplicación de medidas de control para mejorar la asepsia en la atención neonatal, mediante un estudio microbiológico de un Hospital ubicado al sur del Ecuador. Para lo cual se aplicó el método de Inoculación, mediante la técnica de sedimentación en placa, método de aislamiento, método Gravimétrico, Método Volumétrico, método McFarland, se aplicaron pruebas bioquímicas y enzimáticas. Las muestras ambientales (cajas petri) fueron procesadas en el área de bacteriología según el Protocolo de Toma de Muestra de Ambiente, establecido por el Hospital en estudio. Se determinó mediante el estudio microbiológico que la presencia de microorganismos en ambiente del área de neonatología de un Hospital ubicado al Sur del Ecuador fue de $<5$ Unidades Formadores de Colonias por cada muestra positiva analizada y se identificó al S. epidermis como el agente con mayor frecuencia por ostentarse por 4 ocasiones y se identificó a la Unidad de Cuidados Básicos como la unidad con mayor continuidad de colonias por presentar un crecimiento de 11 colonias bacterianas; concluyendo que la presencia de microorganismos en el Hospital estudiado fue mínima. De los 14 muestreos realizados, se destaca S. epidermis en un $80 \%$, S. saprophyticus $12 \%$, E. coli en un 


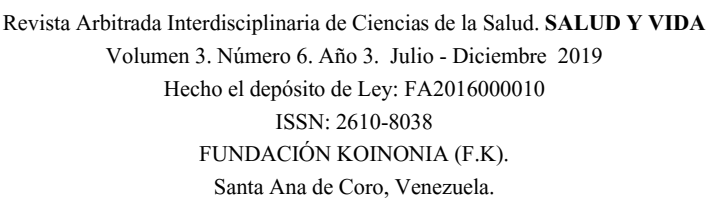

Carmen Silverio Calderón; Cristhian Damian Cabrera Gia

$4 \%$ y Serratia marcescens $4 \%$. Al comparar los resultados obtenidos en las unidades de estudio con la Norma UNE- EN- ISO 14698-1/2:2006 se clasificó como ambientes muy limpios, sin evidenciar riesgo para la salud de los recién nacidos.

Descriptores: Infección Nosocomial; Microorganismos; Calidad Microbiológica Ambiental; Bioseguridad, Recién Nacidos.

\section{ABSTRACT}

This is a descriptive study that meets the objective of identifying the application of control measures to improve asepsis in neonatal care, through a microbiological study of a Hospital located in southern Ecuador. For which the Inoculation method was applied, using the plate sedimentation technique, isolation method, Gravimetric method, Volumetric method, McFarland method, biochemical and enzymatic tests were applied. The environmental samples (petri dishes) were processed in the area of bacteriology according to the Environment Sampling Protocol, established by the Hospital under study. It was determined through the microbiological study that the presence of microorganisms in the environment of the neonatology area of a Hospital located in the South of Ecuador was $<5$ Colony Forming Units for each positive sample analyzed and $\mathrm{S}$. epidermis was identified as the agent with the highest frequency for showing up on 4 occasions and the Basic Care Unit was identified as the unit with the greatest continuity of colonies due to the growth of 11 bacterial colonies; concluding that the presence of microorganisms in the Hospital studied was minimal. Of the 14 samples performed, S. epidermis stands out in $80 \%$, S. saprophyticus $12 \%$, E. coli in $4 \%$ and Serratia marcescens $4 \%$. When comparing the results obtained in the study units with the UNE - EN - ISO 14698- 1/2: 2006 Standard, it was classified as very clean environments, without showing any risk to the health of newborns.

Descriptors: Nosocomial infection; Microorganisms; Environmental Microbiological Quality; Biosecurity, Newborns.

\section{INTRODUCCIÓN}

Las infecciones producidas por bacterias y hongos forman una causa significativa de morbimortalidad a nivel mundial debido a que los neonatos que tienen infección son transportadores de microorganismos patógenos y focos viables de infección para los demás niños y para el personal de salud.

En Ecuador la tasa de mortalidad por infecciones nosocomiales (neumonía nosocomial y bacteriemia) es sumamente alta, aproximadamente 1000 
hospitalizados fallecen por esta razón, a diferencia que, en otros países como Brasil o Perú, pero su incidencia y lugar de mayor prolongación (cuidados intensivos) de contaminaciones es semejante.

De tal manera que el medio ambiente hospitalario contiene considerables microorganismos, pero sólo en algunos casos se ha confirmado claramente una relación causa-efecto entre la apariencia de microorganismos en este medio y el tratamiento de infección en humanos. Los patógenos para los que existe mayor certeza de su capacidad de mantenerse en depósitos ambientales son Clostridium difficile, enterococo, incluyendo los tenaces a la vancomicina, y Staphylococcus aureus, incluyendo los resistentes a la meticilina.

En tal sentido, el ambiente hospitalario puede llegar a hacer un reservorio y fuente de contaminación para pacientes ambulatorios, hospitalizados y sobre todo recién nacidos cuando no se consideran los protocolos de desinfección para la prevención de infecciones intrahospitalarias originadas por microorganismos patógenos. (1)

Ahora bien, es fundamental conocer el concepto de Bioseguridad el cual se define como una doctrina del comportamiento que compromete a todas las personas del ambiente asistencial a diseñar estrategias que disminuyan los riesgos de contaminación. (2)

Se puede manifestar que en el Ecuador este concepto no es muy conocido, por lo que cada día los pacientes se ven afectados por enfermedades nosocomiales que podrían ser evadidas si se vigilara el desempeño riguroso de las normas de Bioseguridad en el hospital.

Hoy en día la presencia excesiva microorganismos patógenos en los hospitales según el área de pacientes inmunocomprometidos son causantes de un sinnúmero de defunciones en la fase neonatal a nivel mundial, actualmente mueren entre 2 y 5 millones de neonatos en sus primeros días de nacidos, aproximadamente 8000 recién nacidos al día, lo que trae consigo un mayor gasto social y económico para las entidades de salud.

Lo anteriormente expuesto, nos conlleva a manifestar que la carga de morbilidad imputable a las infecciones agrupadas a la atención hospitalaria es enorme, según 
información obtenida: en el mundo, millones de pacientes resultan afectados cada año. Estas infecciones causan muertes, discapacidades, y propician la resistencia al antibiótico. (3)

En algunos países, más de la mitad de R.N. ingresados en unidades de neonatología contraen infecciones asociadas a la atención hospitalaria, con una tasa de letalidad comprendida entre el $12 \%$ y el $52 \%$. (4)

Los recién nacidos están expuestos a muchas enfermedades comparado con niños de 9 años o adultos de 25 años de edad, esto se debe a que el sistema inmunitario de los recién nacidos no se encuentra desarrollado ni preparado para combatir ninguna clase de infección. (5) (6)

El estudio microbiológico del ambiente en las diferentes áreas de los hospitales es de vital importancia para tener un control de la presencia o ausencia de agentes etiológicos en las superficies y así reducir los índices de contaminación por gérmenes patógenos que se transportan mediante el aire, flotando sobre moléculas inertes, como: gotas, polvo, partículas etc. (6) (7)

Por lo tanto, es trascendente la prevención de las infecciones a este nivel, para proveer el desarrollo de una microflora inofensiva que disminuya la transmisión de patógenos activos y se atesore la vida, se restaure la salud y se garantice la seguridad del paciente.

En este orden de ideas, el presente estudio realizado en el área de Neonatología del Hospital ubicado al sur de Ecuador, nos dio la oportunidad de abordar un tema tan importante, cuyo objetivo es identificar la aplicación de medidas de control para mejorar la asepsia en la atención neonatal, mediante un estudio microbiológico.

\section{METODOLOGÍA}

El método empleado para esta investigación fue el Método de Inoculación, se aplicó a través de la técnica de sedimentación en placa, el método de aislamiento, método gravimétrico, método volumétrico, método McFarland: Permite obtener una concentración homogénea. (8)

Además de efectuarse varias pruebas bioquímicas de identificación, pruebas enzimáticas, de vital importancia para el aislamiento, y caracterización de las 
bacterias. Para Stafilococcus se realizó: Prueba catalasa, coagulase, oxidasa, fermentación de manitol y susceptibilidad a la novobiocina. Para Enterobacterias se realizó: Prueba de citrato, prueba de ureasa, prueba lisina hierro, prueba hierro triple azúcar, prueba de Indol y motilidad. (9) (10) (11)

\section{Materiales}

Se usó autoclave para la esterilización de los materiales de laboratorio y medios de cultivo. Además, se hizo usó de cajas Petri con medios de cultivos estériles para la toma de muestra, aislamiento de las colonias, para realizar pruebas de susceptibilidad y para identificación de microorganismos. Una incubadora para el desarrollo y almacenamiento de colonias bacterianas a una temperatura de $370 \mathrm{C}$. Se empleó un microscopio para la observación de preparación en fresco y tinción de Gram. Para evitar contaminación se trabajó en la cámara de flujo laminar. Se utilizó una balanza analítica, cocineta, contador de colonias, centrifuga y equipo de baño maría. (12)

\section{Sustancias y reactivos}

Agua destilada, agua oxigenada, solución salina, alcohol etílico, medios de cultivo estériles (Agar Sangre, Agar Macconkey, Agar Mueller Hinton y Agar Manitol Salado), reactivos para tinción de Gram y Discos de Novobiocina 5 mg.

\section{Toma de muestra ambiental}

El muestreo ambiental se realizó dos veces al mes, en horarios de 12:30 p.m. a 13:30 p.m. a las diferentes unidades del Área de Neonatología del Hospital. Para la toma de muestra, se utilizó cajas Petri con medio de cultivo estéril (agar sangre), que fueron colocadas abiertas de 20 a 40 minutos en los diferentes puntos de muestreo (Técnica sedimentación en placa), luego se cerraron las cajas y rápidamente fueron retiradas por el encargado correspondiente y llevadas al laboratorio de Microbiología en una caja transportadora totalmente estéril. (13) (14) 


\section{Procesamiento de la muestra ambiental}

Por otro lado, para la recolección de las muestras, se procede a incubar a $35^{\circ} \mathrm{C}$ hasta 24 horas, se efectuó la lectura y al evidenciar presencia de colonias bacterianas inmediatamente se realizó un aislamiento en medio de cultivo agar sangre y agar Macconkey de las colonias, se incubó al mismo tiempo y temperatura. Según el desarrollo bacteriano de las colonias en los medios de cultivo se realizó una preparación en fresco y tinción de Gram, seguido de las pruebas bioquímicas de identificación para Staphylococcus spp. y para Enterobacterias. (15) (16)

Las muestras ambientales (cajas Petri) fueron procesadas en el área de bacteriología según el Protocolo de Toma de Muestra de Ambiente, establecido por el Hospital en estudio.

\section{RESULTADOS Y DISCUSIÓN}

Tabla 1. Género y/o especies bacterianas aisladas en el Área de Neonatología.

\begin{tabular}{lll}
\hline Género y/o especie bacteriana & n cepas total & \% total \\
\hline Staphylococcus epidermidis & 20 & 80 \\
Staphylococcus saprophyticus & 3 & 12 \\
Escherichia coli & 1 & 4 \\
Serratia marcescens & 1 & 4 \\
\hline Total n (\%) & 25 & $(100)$ \\
\hline
\end{tabular}

Tabla 2. Microorganismos identificados según las diferentes unidades del Área de Neonatología.

\begin{tabular}{llll}
\hline Microorganismos & $\begin{array}{l}\text { Unidad de Cuidados } \\
\text { Básicos }\end{array}$ & $\begin{array}{l}\text { Unidad de Cuidados } \\
\text { Intensivos }\end{array}$ & $\begin{array}{l}\text { Aislamiento } \\
\text { Neonatos }\end{array}$ \\
\hline S. epidermidis & 8 & 5 & 7 \\
S. saprophyticus & 1 & - & 2 \\
E. coli & 1 & - & - \\
Serratia marcescens & 1 & - & - \\
\hline Total n (\%) & $11(52)$ & $5(15)$ & $9(33)$ \\
\hline
\end{tabular}

Los microorganismos identificados en el presente estudio fueron 25 colonias bacterianas, donde los agentes que presentaron índice de mayor frecuencia fueron Staphylococcus epidermidis (20 colonias), S. saprophyticus ( 3 colonias), E. coli (1 
colonia), y Serratia marcescens (1 colonia), siendo estos diferentes a los aislados por Zaragoza, Duany Lourdes, García Heladia, Martínez Ángeles, Peregrino Leoncio y Estuardo Salgado.

Sin embargo, en otros estudios los microorganismos identificados mantuvieron estrecha relación entre sí con la temperatura y el ambiente que nos rodea, y logran reproducirse de manera rápida cuando existen temperaturas altas. (7) (17)

El sitio identificado con la unidad de mayor continuidad de agentes bacterianos fue la Unidad de Cuidados Básicos por identificarse 11 colonias bacterianas, seguido del área de Aislamiento de Neonatos con 9 colonias bacterianas y la Unidad de Cuidados Intensivos con 5 colonias bacterianas; los microorganismos aislados discrepan con los resultados obtenidos por los autores antes en mención.

En Valencia-España, según información reportada por Zaragoza et al. (4) los microorganismos predominantes en la sala de cuidados intensivos fue P. aeruginosa seguido por E. Coli, como principales causantes de contaminaciones intrahospitalarias, estos resultados coinciden con la bacteria E. coli aislada en el presente estudio, pero difiere en su incidencia.

Duany Lourdes et al. (2005) (18) , reportaron mediante un estudio descriptivo la incidencia de infecciones intrahospitalarias con un índice de 3,2 infantes por cada 100 egresados, identificando a la neumonía como la infección con mayor periodicidad, y a la $P$. aeruginosa con $27,3 \%$ como el agente infeccioso con mayor frecuencia, seguido del Estafilococo coagulasa negativo con 12,3\%, y se identificó como consecuencia principal a la canalización venosa seguido del mal uso de los antibióticos que a diferencia del presente estudio efectuado en un Hospital ubicado al Sur del Ecuador no se identificó factores de riesgo intrínseco y extrínseco, pero el estudio de Danny Lourdes se asemeja con el estudio ejecutado por Zaragoza indicando a la $P$. aeruginosa como el principal agente patógeno causante de infecciones intrahospitalarias a nivel neonatal.

Miriam Pugo-Luisa Reinoso (2010) (19), por medio de su estudio descriptivo concluyeron que la determinación de carga microbiana encontrada fue en los sitios de mayor número de colonias: las manos del personal de enfermería, termómetros y hoods con un crecimiento de más de 50 colonias de coliformes. Para lo cual, 
pudieron determinar que el personal de enfermería no aplica medidas de asepsia inmediatas dentro del área de Neonatología, empezando por la aplicación de la técnica adecuada del lavado de manos, la correcta clasificación de desechos, la desinfección adecuada de las termocunas y equipos utilizados en el área.

Un estudio microbiológico realizado por Estuardo Salgado en Quito en marzo del 2017 (20), identificó a la Pseudomona aeruginosa y Acinetobacter baumanni como los principales agentes patógenos causantes de infecciones intrahospitalarias y se estima aproximadamente 20 neumonías nosocomiales por cada 1000 días.

En Ecuador según datos de la Comunidad Científica Internacional de Control de Infecciones Nosocomiales mencionan que nuestro país está por arriba del 50\% por poseer mayor índice de mortalidad por contaminaciones hospitalarias siendo la causa principal la neumonía y el mal uso de antibióticos, de los 43 países confederados.

De acuerdo con el estudio microbiológico realizado, los resultados manifestaron que los gérmenes identificados en las diferentes unidades del área de neonatología, no presumen riesgo para la salud de los recién nacidos ni para el personal que labora en las diferentes unidades, debido a que los recuentos bacterianos se encuentran dentro del rango permisible según la norma UNE-EN-ISO 14698-1/2:2006 considerando como AMBIENTE MUY LIMPIO, cuando el recuento microbiológico es de $<10$ UFC/m3 en salas de Neonatología y Quirófano, sin embargo es de vital importancia cumplir los protocolos de asepsia correctamente para restringir posibles indisposiciones en la salud.

\section{CONCLUSIÓN}

Se concluyó mediante el estudio microbiológico que la presencia de microorganismos en ambiente del área de neonatología de un Hospital ubicado al Sur del Ecuador fue de $<5$ Unidades Formadores de Colonias por cada muestra positiva analizada y se identificó al $S$. epidermidis como el agente con mayor frecuencia por ostentarse por 4 ocasiones y se identificó a la Unidad de Cuidados Básicos como la unidad con mayor continuidad de colonias por presentar un 
crecimiento de 11 colonias bacterianas; concluyendo que la presencia de microorganismos en el Hospital estudiado fue mínima.

\section{REFERENCIAS CONSULTADAS}

1. Belizón, Y. E., Aguilera, J. C., Pompa, J. A., \& García, A. L. (agosto de 2013). Factores de riesgo de infección intrahospitalaria en pacientes ingresados en unidades de cuidados intensivos. MEDISAN, (17) 3070-3071.

2. Bioseguridad. Definición de bioseguridad. Julio 2006 disponible en: http://bioseguridadutem.blogspot.com/2006/07/(2)definicindebioseguridad.html

3. Bou, G., Fernández Olmos, A., García, C., Sáez Nieto, J., \& Valdezate, S. (marzo de 2014). Frecuencia de aislamientos microbiológicos y perfil de resistencia bacteriana en 13 clínicas y hospitales de alta complejidad en Santiago de Cali - Colombia. Infectio, 18(16), 3-11. doi:https://doi.org/10.1016/S0123-9392(14)70734-9

4. Cerero, L. L. (agosto - septiembre de 2014). Papel del ambiente hospitalario y los equipamientos en la transmisión de las infecciones nosocomiales. Enfermedades Infecciosas y Microbiología Clínica, 32(6), 459-464. doi: 10.1016/j.eimc.2013.10.004

5. Delgado, M., Muñoz, A., Orejuela, L., \& Sierra, C. (2003). Algunos factores de riesgo para mortalidad neonatal en un hospital de III nivel. Colombia Médica, 34(15), 179-185. Obtenido de http://www.redalyc.org/articulo.oa?id=28334402

6. Duany Badell, L. E., Losa Pérez, D., Ávila Ramírez, M., Barletta del Castillo, J. E., Hernández Malpica, S., \& Gómez Morejón, A. (junio de 2014). Caracterización de la infección nosocomial en una unidad de cuidados intensivos pediátricos. Cienfuegos 2005-2009. Medisur, 12(18), 462469. Obtenido de http://scielo.sld.cu/scielo.php?script=sci arttext\&pid=S1727$\underline{897 \times 2014000300002}$

7. García, H., Martínez Muñoz, Á. N., \& Peregrino Bejarano, L. (7 de abril de 2014). Epidemiología de las infecciones nosocomiales en una unidad de cuidados intensivos neonatales. Revista Médica del Instituto Mexicano del Seguro Social, 52(14), 30-37. Obtenido de http://www.redalyc.org/articulo.oa?id=457745486006 
8. Macas Poma, G. (2017). PROTOCOLO PARA TOMA DE MUESTRA AMBIENTAL. Protocoló de toma de muestra ambiental, H.O.A.L.O., Laboratorio Clínico, Santa Rosa. (13)

9. Méndez Puentes, C., Camacho Suárez, J., \& Echeverry Hernández, S. (Julio de 2015). Identificación de bacterias y hongos en el aire de Neiva, Colombia. Rev. salud pública, 17(5), 728-737. doi:https://doi.org/10.15446/rsap. v17n5.38468

10. Menis Ferreira, A., de Andrade, D., Rigotti, M., Gotardo de Almeida, M., García Guerra, O., \& García, A. (junio de 2015). Evaluación de la desinfección de superficies hospitalarias por diferentes métodos de monitorización. Rev. Latino-Am. Enfermagem, 23(9), 466-474. doi:10.1590/01041169.0094.2577

11. Miriam Pugo., Luisa Reinoso (2010) (19) Tesis de grado "Aplicación De Las Medidas De Bioseguridad En La Atención De Enfermería Al Neonato En El Servicio De Neonatología Del Hospital Vicente Corral Moscoso Cuenca 2009 - 2010."

12. Programa de infecciones nosocomiales. Dr. Nelson Vásconez MD. Ms. Ecuador agosto 2007. (4)

13. Repáraz Federico. Limpieza y desinfección en el hospital. Disponible en: www.cfnavarra.es/salud/anales/textos/vol23/suple2/suple8.html (3)

14. Rodríguez Ocaña, E. (17 de enero de 2017). Tifus y laboratorio en la España de posguerra. Revista Dynamis, 37(1), 489-515. Obtenido de http://scielo.isciii.es/scielo.php?script=sci arttext\&pid=S0211$\underline{95362017000200011}$

15. Romero Bohórquez, C., Fernando Castañeda, D., \& Acosta Peñaloza, G. (diciembre de 2016). Determinación de la calidad bacteriológica del aire en un laboratorio de microbiología en la Universidad Distrital Francisco José de Caldas en Bogotá, Colombia. NOVA, 14(11), 103-111. Obtenido de http://www.scielo.org.co/scielo.php?script=sci arttext\&pid=S17942470201600 0200012\&lng=en.

16. Toloza, D. L., \& Lizarazo, L. M. (2013). Calidad Microbiológica Del Ambiente De La Biblioteca Alfonso Patiño Rosselli, Tunja Boyacá (Colombia). Revista U.D.C.A Actualidad \& Divulgación Científica, (10) 43-52.

17. Vargas Flores, T., \& Villazante Condori, L. G. (2014). Clasificación de los Microorganismos. Revista de actualización Clínica, 44 (12), 2309-2313. Vizcarra Munguía, V., Anaya González, L., Villarreal Treviño, P., \& Cuello 
García, C. (2011). Factores de riesgo asociados a infección nosocomial en unidades de cuidados intensivos neonatales: Perspectiva de Seguridad del Paciente. Revista CONAMED, 16(9), 11-21. Obtenido de http://www.dgdiconamed.salud.gob.mx/ojsconamed/index.php/revconamed/ar ticle/view/344

18.Zamora, E. J., Masaquiza, D. A., Moreno, F. A., \& Gutiérrez, E. R. (2018). La infección nosocomial. Un reto en las unidades de cuidados intensivos. Enfermería Investiga, 30-31. (8)

19.Zaragoza, R., Ramírez, P., \& López Pueyo, M. J. (mayo de 2014). Infección nosocomial en las unidades de cuidados intensivos. Enfermedades Infecciosas y Microbiología Clínica., 32(7), 320-327. doi: 10.1016/j.eimc.2014.02.006

20. https://www.redaccionmedica.ec/secciones/salud-publica/ecuador-con-lamayor-tasa-de-mortalidad-por-infecciones-nosocomiales-89901 (20)

\section{REFERENCES CONSULTED}

1. Belizón, Y. E., Aguilera, J. C., Pompa, J. A., \& García, A. L. (August 2013). Risk factors for in-hospital infection in patients admitted to intensive care units. MEDISAN, (17) 3070-3071.

2. Biosecurity. Biosafety definition. July 2006 available at: http://bioseguridadutem.blogspot.com/2006/07/(2)definicindebioseguridad.html

3. Bou, G., Fernández Olmos, A., García, C., Sáez Nieto, J., \& Valdezate, S. (March 2014). Frequency of microbiological isolates and bacterial resistance profile in 13 highly complex clinics and hospitals in Santiago de Cali Colombia. Infectio, 18 (16), 3-11. doi: https: //doi.org/10.1016/S0123-9392 (14) 70734-9

4. Cerero, L. L. (August - September 2014). Role of the hospital environment and equipment in the transmission of nosocomial infections. Infectious Diseases and Clinical Microbiology, 32 (6), 459-464. doi: 10.1016 / j.eimc. 2013.10.004

5. Delgado, M., Muñoz, A., Orejuela, L., \& Sierra, C. (2003). Some risk factors for neonatal mortality in a III level hospital. Medical Colombia, 34 (15), 179-185. Retrieved from http://www.redalyc.org/articulo.oa?id=28334402

6. Duany Badell, L. E., Losa Pérez, D., Ávila Ramírez, M., Barletta del Castillo, J. E., Hernández Malpica, S., \& Gómez Morejón, A. (June 2014). 
Characterization of nosocomial infection in a pediatric intensive care unit. Cienfuegos 2005-2009. Medisur, 12 (18), 462-469. Retrieved from http://scielo.sld.cu/scielo.php?script=sci arttext\&pid=S1727$\underline{897 \times 2014000300002}$

7. García, H., Martínez Muñoz, Á. N., \& Peregrino Bejarano, L. (April 7, 2014). Epidemiology of nosocomial infections in a neonatal intensive care unit. Medical Journal of the Mexican Social Security Institute, 52 (14), 30-37. Retrieved from http://www.redalyc.org/articulo. oa? id=457745486006

8. Macas Poma, G. (2017). PROTOCOL FOR ENVIRONMENTAL SAMPLING. Environmental sampling protocol, H.O.A.L.O., Clinical Laboratory, Santa Rosa. (13)

9. Méndez Puentes, C., Camacho Suárez, J., \& Echeverry Hernández, S. (July 2015). Identification of bacteria and fungi in the air of Neiva, Colombia. Rev. public health, 17 (5), 728-737. doi: https: //doi.org/10.15446/rsap. v17n5.38468

10. Menis Ferreira, A., de Andrade, D., Rigotti, M., Gotardo de Almeida, M., García Guerra, O., \& García, A. (June 2015). Evaluation of the disinfection of hospital surfaces by different monitoring methods. Rev. Latino-Am. Enfermagem, 23 (9), 466-474. doi: 10.1590 / 01041169.0094.2577

11. Miriam Pugo., Luisa Reinoso (2010) (19) Thesis "Application of Biosafety Measures in Nursing Care to the Neonate in the Neonatology Service of Vicente Corral Moscoso Cuenca Hospital 2009 - 2010."

12. Program of nosocomial infections. Dr. Nelson Vásconez MD. Ms. Ecuador August 2007. (4)

13. Repáraz Federico. Cleaning and disinfection in the hospital. Available at: www.cfnavarra.es/salud/anales/textos/vol23/suple2/suple8.html (3)

14. Rodríguez Ocaña, E. (January 17, 2017). Typhus and laboratory in postwar Spain. Dynamis Magazine, 37 (1), 489-515. Retrieved from http://scielo.isciii.es/scielo.php?script=sci arttext\&pid=S0211$\underline{95362017000200011}$

15. Romero Bohórquez, C., Fernando Castañeda, D., \& Acosta Peñaloza, G. (December 2016). Determination of the bacteriological quality of the air in a microbiology laboratory at the Francisco José de Caldas District University in Bogotá, Colombia. NOVA, 14 (11), 103-111. Retrieved from http://www.scielo.org.co/scielo.php?script=sci arttext\&pid=S17942470201600 $\underline{0200012 \& \operatorname{lng}=e n \text {. }}$ 
16. Toloza, D. L., \& Lizarazo, L. M. (2013). Microbiological Quality of the Environment of the Alfonso Patiño Rosselli Library, Tunja Boyacá (Colombia). U.D.C.A Magazine News \& Scientific Dissemination, (10) 43-52.

17. Vargas Flores, T., \& Villazante Condori, L. G. (2014). Classification of Microorganisms. Clinical Update Magazine, 44 (12), 2309-2313. Vizcarra Munguía, V., Anaya González, L., Villarreal Treviño, P., \& Cuello García, C. (2011). Risk factors associated with nosocomial infection in neonatal intensive care units: Patient Safety Perspective. CONAMED Magazine, 16 (9), 11-21. Retrieved from http://www.dgdiconamed.salud.gob.mx/ojsconamed/index.php/revconamed/art icle/view/344

18.Zamora, E. J., Masaquiza, D. A., Moreno, F. A., \& Gutiérrez, E. R. (2018). Nosocomial infection $A$ challenge in intensive care units. Nursing Research, 30-31. (8)

19.Zaragoza, R., Ramírez, P., \& López Pueyo, M. J. (May 2014). Nosocomial infection in intensive care units. Infectious Diseases and Clinical Microbiology., 32 (7), 320-327. doi: 10.1016 / j.eimc. 2014.02.006

20. https://www.redaccionmedica.ec/secciones/salud-publica/ecuador-con-lamayor-tasa-de-mortalidad-por-infecciones-nosocomiales-89901 (20)

(C2019 por los autores. Este artículo es de acceso abierto y distribuido según los términos y condiciones de la licencia Creative Commons Atribución-NoComercial-Compartirlgual 4.0 Internacional (CC BY-NC-SA 4.0) (https://creativecommons.org/licenses/by-nc-sa/4.0/). 